\title{
Radiologically Unifocal Invasive Breast Carcinomas: Large-Section Histopathology Correlate and Impact on Surgical Management
}

Tibor Tot* and Maria Gere

Department of Pathology and Clinical Cytology, Falun County Hospital, Falun, Sweden

\begin{abstract}
Background: Most breast carcinomas are morphologically complex, comprising both in situ and invasive components that can be unifocal, multifocal, or diffuse. Pre-operative radiological mapping often reveals this complexity, but even in the era of modern multimodality breast imaging lesions may remain undetected.

Methods: We studied the sub gross morphology of tumors in a series of invasive carcinomas determined to be unifocal on pre-operative multimodal radiological imaging. We focused on histological outcome, margin status, and type of surgery. All of the surgical specimens were documented in large-format histological slides.

Results: A total of $44.5 \%$ (344/773) of the tumors had separate invasive and/or in situ foci in large-format histopathology slides, in addition to the radiologically detected tumor focus. The foci occupied an area $\geq 40 \mathrm{~mm}$ in $29.0 \%$ (224/773) of the cases, indicating extensive disease. Close/dirty margin upon histological examination was associated with extensive disease $(32.1 \%$ vs $5.6 \%$, RR $5.6924, p<0.0001)$, multifocality of the invasive component ( $26.0 \%$ vs $3.3 \%, R R=7.9755, p=0.0001)$, and breast conservation $(15.4 \%$ vs $6.3 \%, R R=2.4476, p=0.0036)$, but the differences were found only for extensive tumors. Mastectomy was chosen as the primary or complete intervention in $28.3 \%$ (219/773) of cases, mostly in multifocal and extensive tumors.
\end{abstract}

Conclusion: Our results indicate the presence of extensive disease in a considerable number of breast cancer cases judged pre-operatively as unifocal. Removing the radiologically detectable tumor focus with "no ink on the tumor" may not be sufficient in such cases.

Keywords: Breast; Unifocal tumor; Multifocality; Disease extent; Mastectomy

\section{Introduction}

Most breast carcinomas are morphologically complex lesions comprising both in situ and invasive components, which can be unifocal, multifocal, or diffuse. This complexity is best evidenced in cases with a detailed systematic radiological-pathological correlation [1] documented in large-format histological slides [2]. Figure 1 illustrates the 17 basic aggregate growth patterns of the in situ and invasive tumor components in a series of 1000 breast carcinoma cases and shows that real unifocal lesions represent a minority of breast carcinomas, whereas a considerable proportion of breast cancers are multifocal or diffuses [2].

Modern diagnostic breast imaging methods have high sensitivity and specificity and can accurately map the disease pre-operatively and guide the therapeutic decision making, especially if combined with a multimodal approach [3]. The imaging methods are less sensitive and specific with small invasive tumor foci [4] and non-calcified in situ foci [5], although whole breast ultrasound, tomosynthesis, and magnetic resonance imaging (MRI) perform better than mammography in this aspect $[4,6,7]$.

The recent trend of more and more restrictive surgery in breast cancer is based on studies showing the same outcome in patients treated with breast conservation and irradiation as in patients treated with mastectomy. Some studies have indicated no further benefit of margins wider than "no tumor on ink" in terms of local disease control [8], even if the tumors are multifocal $[9,10]$. On the other hand, studies indicating more unfavorable outcomes in multifocal and extensive carcinomas compared to unifocal carcinomas and those with limited extent have also been published [11-13].

We analyzed the findings of large-format histology slides in cases of breast cancers judged to be unifocal based on pre-operative radiological mapping. We focused on histological outcome, margin status, and type of surgery. In particular, we were interested in the proportion of radiologically occult extensive disease and its impact on surgical management.

\section{Methods}

\section{Patient selection}

We studied a consecutive series of 1663 invasive breast carcinoma cases diagnosed at the Department of Pathology and Clinical Cytology at County Hospital Dalarna in Falun, Sweden, between January 2008 and June 2015. We excluded all recurrences of tumors diagnosed prior to the study period $(\mathrm{n}=120)$ and cases that received any type of neoadjuvant therapy $(\mathrm{n}=39)$. Of the remaining 1504 cases, $1403 \mathrm{had}$ complete radiology data in our database. A total of 843 cases were radiologically unifocal based on pre-operative disease mapping, 773 of which had invasive cancer upon postoperative histological examination. Microinvasive tumors $(<1 \mathrm{~mm}$ in size) were not included. These 773 patients comprised the study cohort. The study was part of a larger

*Corresponding author: Tot T, Department of Pathology and Clinical Cytology Falun County Hospital, 79182 Falun, Sweden, Tel: 004623492696; Fax: 004623492389; E-mail: tibor.tot@Itdalarna.se

Received January 11, 2016; Accepted March 04, 2016; Published March 07 2016

Citation: Tot T, Gere M (2016) Radiologically Unifocal Invasive Breast Carcinomas: Large-Section Histopathology Correlate and Impact on Surgical Management. J Cancer Sci Ther 8: 050-054. doi:10.4172/1948-5956.1000389

Copyright: (c) 2016 Tot T, et al. This is an open-access article distributed under the terms of the Creative Commons Attribution License, which permits unrestricted use, distribution, and reproduction in any medium, provided the original author and source are credited. 
Citation: Tot T, Gere M (2016) Radiologically Unifocal Invasive Breast Carcinomas: Large-Section Histopathology Correlate and Impact on Surgical Management. J Cancer Sci Ther 8: 050-054. doi:10.4172/1948-5956.1000389

project approved by the Regional Ethical Committee Uppsala/Örebro. Written consent was obtained from the patients.

\section{Pre-operative radiological work-up}

The pre-operative radiological work-up comprised a clinical examination, mammography, and ultrasound examination in all cases. Magnetic resonance imaging (MRI) was carried out in 75\% (581/773) of the cases. The radiologists reported their findings to the pathology department on a data sheath prior to a multidisciplinary tumor board and the data were registered in the pathology research database. The radiological diagnosis was confirmed by core-needle biopsy (754 cases) or fine-needle aspiration biopsy (12 cases) in all but seven cases. All of the cases were discussed at the multidisciplinary tumor board before and after the surgical intervention.

\section{Surgery}

Breast conserving surgery (sectorial resection) with sentinel lymph node biopsy was the standard procedure for unifocal breast carcinomas in our institution and carried out in 538 cases in the present series. Modified radical mastectomy was indicated at the patient's request or on the basis of a large tumor size. Axillary lymph node clearance was performed in 55 cases. A total of 481 patients underwent postoperative irradiation.

The completeness of the surgical intervention was assessed by the radiologists based on routine intraoperative specimen radiography. No histopathological examination was carried out intraoperatively with frozen sections or other methods to assess the specimen margins. During the histological evaluation, we followed the recommendations of the regional guidelines of Uppsala/Örebro [14] recommending sectorial resection for tumors $<4 \mathrm{~cm}$ with $10 \mathrm{~mm}$ minimal macroscopic margin at the beginning of the study period and "no tumor on the ink" at histological evaluation since April 2011. Immediate complete surgery was indicated if a close/dirty margin was seen upon intraoperative radiological analysis of the specimen. Complete mastectomy followed histopathologically established close or dirty margin(s).

\section{Histopathological method and parameters}

All specimens were prepared using the large-format histopathology method performed routinely in our laboratory since 1982 and described in detail elsewhere [15]. Sector-resection specimens were sliced into 3 to $4 \mathrm{~mm}$ thick tissue slices parallel to the pectoralis fascia and subjected to radiography. One to five of the most representative slices (measuring up to $9 \mathrm{~cm} \times 8 \mathrm{~cm}$ ) were selected and embedded in large paraffin blocks. Larger slices were bisected and embedded into separate blocks. Mastectomy specimens were sliced perpendicular to the pectoralis fascia to visualize the surgical margin on one histological level. Slices were inked to keep the orientation of the specimen.

The distributions of the invasive and in situ components from the same lesion were determined separately using previously published criteria $[2,15,16]$. Unifocal invasive tumors comprised a single well-delineated focus on the large histology slide. Invasive tumor multifocality was defined as the presence of more than one welldelineated invasive tumor focus with non-malignant tissue or in situ tumor in between the foci. Diffuse invasive tumors were identified as tumors dispersed over a large area of the section, similar to a spider web, with no distinct tumor mass. The in situ components of the tumors were regarded as "unifocal" if involving a single terminal ductal lobular unit or several neighboring terminal units without uninvolved breast tissue in between, as "multifocal" if involving several distant terminal ductal lobular units with uninvolved breast tissue in between, and as "diffuse" if involving mainly the larger ducts. If a complete surgical intervention was performed in addition to the primary sector resection, an attempt was made to summarize the findings for the entirety of the excised tissue. The aggregate growth patterns of in situ and invasive tumor components are illustrated in Figure 1. A typical case is illustrated in Figure 2.

Tumor size was defined as the largest dimension of the largest invasive focus. Disease extent was defined as the tissue area containing all in situ and invasive malignant structures and measured in two dimensions. Cases in which the tumor structures occupied an area at

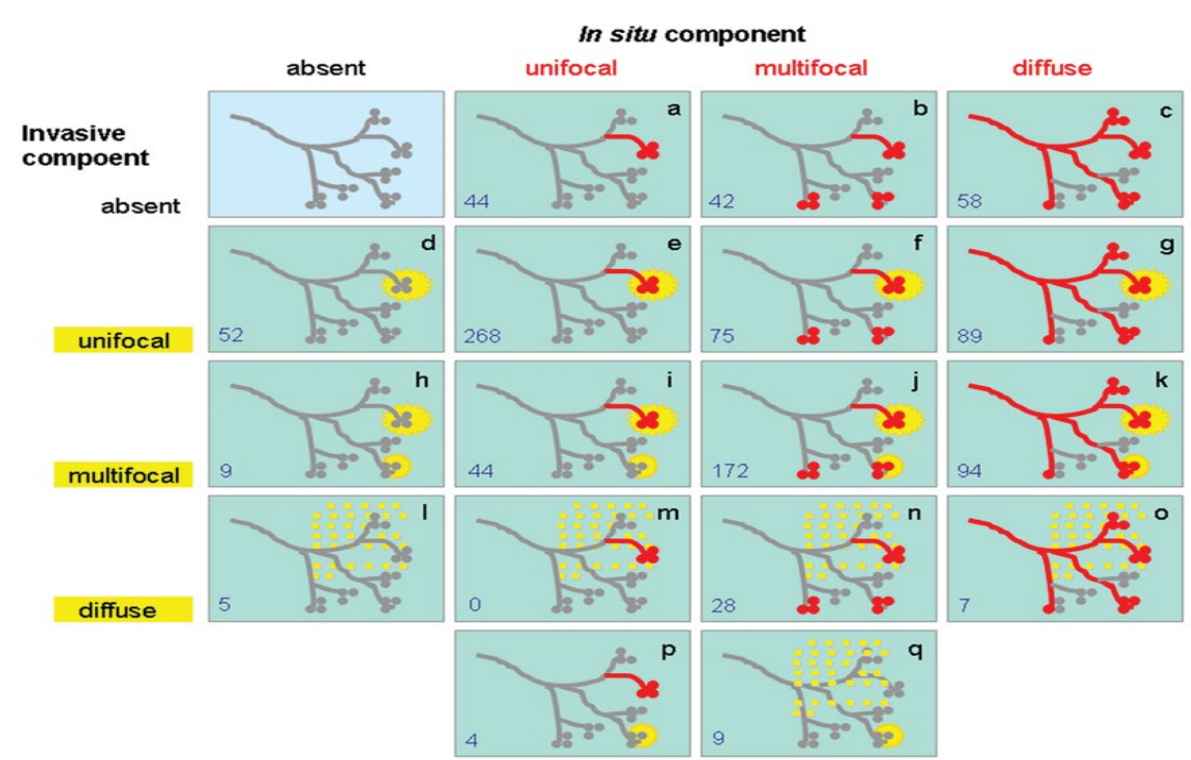

Figure 1: Possible combined growth patterns in breast carcinomas. Tumors with unifocal (d, e, f, g, p), multifocal (h, i, j, k), and diffuse (l, m, n, o, q) invasive component, and with unifocal $(a, e, i, m, p)$, multifocal $(b, f, j, n)$, and diffuse $(c, g, k, o)$ in situ component are illustrated. Numbers in the lower left corner of the drawings indicate the number of cases in the series of 1,000 consecutive breast carcinomas belonging to that category (Reprinted from Ref. 2 , open access publication). 


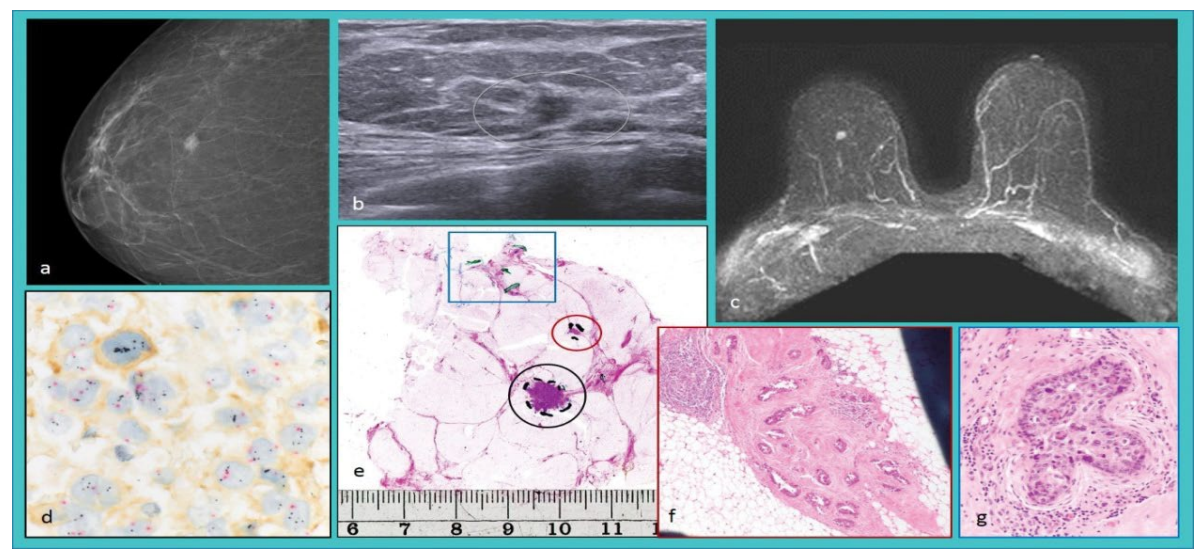

Figure 2: Radiologicallyunifocal invasive breast carcinoma. (a) Radiogram, (b) Sonogram, and (c) Magnetic resonance image. (d) Tricolor bright field in situ hybridization showing HER2 gene amplification. (e) Large-format histology of the surgical specimen shows the radiologically detected invasive tumor focus (black circle), an additional invasive focus (red circle), and structures of the in situ carcinoma (blue box). (f) Magnification of the additional invasive focus and (g) in situ component stained with hematoxylin and eosin.

least $40 \mathrm{~mm}$ in its largest dimension was categorized as extensive and other cases as non-extensive.

\section{Statistical analysis}

Comparisons of proportions with the chi-square test and relative risk assessment were carried out using the program MedCalc Statistics for Biomedical Research (MedCalc Software, Belgium). P-values $<0.05$ were considered significant.

\section{Results}

The radiological tumor size estimated on the basis of mammography and ultrasound measurements was $1-9 \mathrm{~mm}$ in $28.7 \%(222 / 773)$ of cases, $10-19 \mathrm{~mm}$ in $44.4 \%$ (343/773), $20-29 \mathrm{~mm}$ in $16.2 \%$ (125/773), $30-39 \mathrm{~mm}$ in $6.3 \%(49 / 773)$, and $40 \mathrm{~mm}$ or larger in $3.6 \%(28 / 773)$. In six cases the radiological size was unknown. The histological size of the radiologically detected tumor focus was $1-9 \mathrm{~mm}$ in $16.9 \%(131 / 773)$ of cases, $10-19 \mathrm{~mm}$ in $51.9 \%$ (401/773), $20-29 \mathrm{~mm}$ in $18.6 \%(144 / 773)$, $30-39 \mathrm{~mm}$ in $8.0 \%(62 / 773)$, and $40 \mathrm{~mm}$ or larger in $4.5 \%$ (35/773). All diffuse invasive carcinomas were larger than $20 \mathrm{~mm}$, and $52.0 \%(14 / 27)$ measured $\geq 40 \mathrm{~mm}$.

Of the tumors characterized radiologically as unifocal, $44.5 \%$ (344/773) had separate invasive and/or in situ foci in large-format histopathology slides. The non-unifocal tumors were categorized as cases with a unifocal invasive focus and multifocal or diffuse in situ component, cases with a multifocal invasive component with any in situ component, or cases with a diffuse invasive component and any in situ component, comprising 22.1\% (171/773), 18.9\% (146/773), and $3.5 \%(27 / 773)$ of the whole series, respectively. Of the 146 cases with multiple invasive foci, 60 had more than one additional invasive focus. The size of the additional invasive foci was $1-9 \mathrm{~mm}$ in $93.2 \%$ $(136 / 146)$ of cases, $10-14 \mathrm{~mm}$ in $6.1 \%(9 / 146)$, and $>20 \mathrm{~mm}$ in one case. Among unifocal invasive tumors associated with a non-unifocal in situ component, the in situ component was multifocal in $60.8 \%(104 / 171)$ of cases and diffuses in $39.2 \%(867 / 171)$ of cases. The tumor foci occupied an area $\geq 40 \mathrm{~mm}$ in $29.0 \%(224 / 773)$ of the cases, indicating extensive disease (Table 1).

A close/dirty margin upon histological examination was found in $13.3 \%(103 / 773)$ of cases, more often in extensive disease than in non-extensive disease $(32.1 \%, 72 / 224$ vs $5.6 \%, 31 / 549$; $R R=5.6924$, $\mathrm{p}$
$<0.0001$ ), in cases with a multifocal invasive component compared to those with a unifocal invasive component $(26.0 \%, 38 / 146$ vs $3.3 \%$, 14/429; $\mathrm{RR}=7.9755, \mathrm{p}=0.0001$ ), and in patients who underwent breast conservation compared to those treated with mastectomy (15.4\%, $92 / 598$ vs. $6.3 \%, 11 / 175 ; \mathrm{RR}=2.4476, \mathrm{p}=0.0036$ ). If these results are broken down into morphological subgroups (Table 2), significant differences are seen only in extensive tumors with a multifocal invasive component and in cases with a unifocal invasive and non-unifocal in situ component.

Mastectomy was chosen as the primary or complete intervention in $28.3 \%(219 / 773)$ of cases: $47.8 \%(107 / 224)$ of extensive cases and $20.4 \%(112 / 549)$ of non-extensive cases $(\mathrm{RR}=2.3415, \mathrm{p}<0.0001)$. As seen in Table 1, significantly different mastectomy rates were found between extensive and non-extensive cases among histologically unifocal tumors ("extensive" in this subgroup was related to tumor size $\geq 40 \mathrm{~mm} ; 66.7 \%, 12 / 18$ vs $19.2 \%, 79 / 411, \mathrm{p}<0.0001)$ and tumors with histologically verified multiple invasive foci $(63.0 \%, 51 / 81$ vs $23.1 \%$, $15 / 65, \mathrm{p}<0.0001)$. No such association was found among tumors with histologically unifocal invasive and non-unifocal in situ components $(29.2 \%, 31 / 106$ vs $23.1 \%, 15 / 65, p=0.4948)$ or diffuse invasive breast carcinomas $(68.4 \%, 13 / 19$ vs $37.5 \%, 3 / 8, \mathrm{p}=0.3081)$.

\section{Discussion}

A recent meta-analysis of studies including more than 10,000 women treated with breast conserving surgery found that $35 \%$ of the patients who did not and $19.3 \%$ who did undergo postoperative irradiation had local recurrence during a 10-year follow-up period [17]. This high incidence, especially in non-irradiated patients, may be related to tumor foci that remained undetected in the pre-operative radiological examination and were left behind after a seemingly radical surgical intervention. In this study, we focused on tumors judged as unifocal based on pre-operative multimodality breast imaging and analyzed the frequency of additional radiologically occult malignant lesions in large-format histology slides. We paid special attention to how such foci influenced the histological surgical margin status and the performance of complete surgery.

The extent of the disease, defined as the tissue volume containing all in situ and invasive malignant structures, was the most important parameter related to the defined outcomes in this study; significant differences were only found in extensive disease. These results are 
Citation: Tot T, Gere M (2016) Radiologically Unifocal Invasive Breast Carcinomas: Large-Section Histopathology Correlate and Impact on Surgical Management. J Cancer Sci Ther 8: 050-054. doi:10.4172/1948-5956.1000389

\begin{tabular}{|c|c|c|c|c|c|}
\hline Surgical Treatment & Extensive disease & $\begin{array}{l}\text { Mastectomy rates, } \\
\text { extensive disease }\end{array}$ & $\begin{array}{l}\text { Non-extensive } \\
\text { disease }\end{array}$ & $\begin{array}{l}\text { Mastectomy rates, non-extensive } \\
\text { disease }\end{array}$ & $P$ value \\
\hline $\begin{array}{l}\text { Unifocal invasive cancer without multifocal or } \\
\text { diffuse in situ component }\end{array}$ & $4.2 \%(18 / 429)$ & $66.7 \%(12 / 18)$ & $95.8 \%(411 / 429)$ & $19.2 \%(79 / 411)$ & $p<0.0001$ \\
\hline $\begin{array}{l}\text { Unifocal invasive cancer with multifocal or } \\
\text { diffuse in situ component }\end{array}$ & $62 \%(106 / 171)$ & $29.2 \%(31 / 106)$ & $38 \%(65 / 171)$ & $23.1 \%(15 / 65)$ & $p=0.4948$ \\
\hline $\begin{array}{c}\text { Multifocal invasive cancer, any in situ } \\
\text { component }\end{array}$ & $55.5 \%(81 / 146)$ & $63 \%(51 / 81)$ & $44.5 \%(65 / 146)$ & $23.1 \%(15 / 65)$ & $p<0.0001$ \\
\hline Diffuse invasive cancer, any in situ component & $70 \%(19 / 27)$ & $68.4 \%(13 / 19)$ & $30 \%(8 / 27)$ & $37.5 \%(3 / 8)$ & $p=0.3081$ \\
\hline Total & $29 \%(224 / 773)$ & $47.8 \%(107 / 224)$ & $71 \%(549 / 773)$ & $20.4 \%(112 / 549)$ & $p<0.0001$ \\
\hline
\end{tabular}

Table 1: Proportions of mastectomy cases by lesion distribution and disease extent, Dalarna 2008-15.

\begin{tabular}{|c|c|c|c|c|c|}
\hline Surgical Treatment & $\begin{array}{l}\text { Close/dirty margin, primary } \\
\text { mastectomy }\end{array}$ & $\begin{array}{l}\text { Close/dirty margin, breast } \\
\text { conservation }\end{array}$ & $\begin{array}{l}\text { P value chi } \\
\text { square test }\end{array}$ & $\begin{array}{l}\text { Complete } \\
\text { mastectomy }\end{array}$ & $\begin{array}{l}\text { Mastectomy } \\
\text { total }\end{array}$ \\
\hline \multicolumn{6}{|l|}{ Extensive disease } \\
\hline $\begin{array}{l}\text { Unifocal invasive cancer without multifocal or diffuse } \\
\text { in situ component }\end{array}$ & $0 \%(0 / 11)$ & $14.3 \%(1 / 7)$ & $p=0.8331$ & 1 & $66.7 \%(12 / 18)$ \\
\hline $\begin{array}{l}\text { Unifocal invasive cancer with multifocal or diffuse in } \\
\text { situ component }\end{array}$ & $10.5 \%(2 / 19)$ & $43.7 \%(38 / 87)$ & $p=0.0154$ & 12 & $29.2 \%(31 / 106)$ \\
\hline Multifocal invasive cancer, any in situ component & $17.1 \%(6 / 35)$ & $47.8 \%(22 / 46)$ & $p=0.0076$ & 16 & $63 \%(51 / 81)$ \\
\hline Diffuse invasive cancer, any in situ component & $8.3 \%(1 / 12)$ & $28.6 \%(2 / 7)$ & $p=0.5758$ & 1 & $68.4 \%(13 / 19)$ \\
\hline Total & $11.7 \%(9 / 76)$ & $42.5 \%(63 / 148)$ & $p<0.0001$ & 30 & $47.8 \%(107 / 224)$ \\
\hline \multicolumn{6}{|l|}{ Non-extensive disease } \\
\hline $\begin{array}{l}\text { Unifocal invasive cancer without multifocal or diffuse } \\
\text { in situ component }\end{array}$ & $1.4 \%(1 / 74)$ & $3.6 \%(12 / 337)$ & $p=0.3537$ & 5 & $19.2 \%(79 / 411)$ \\
\hline $\begin{array}{l}\text { Unifocal invasive cancer with multifocal or diffuse in } \\
\text { situ component }\end{array}$ & $0 \%(0 / 11)$ & $14.8 \%(8 / 54)$ & $p=0.3831$ & 4 & $23.1 \%(15 / 65)$ \\
\hline Multifocal invasive cancer, any in situ component & $9.1 \%(1 / 11)$ & $16.7 \%(9 / 54)$ & $p=0.8333$ & 4 & $23.1 \%(15 / 65)$ \\
\hline Diffuse invasive cancer, any in situ component & $0 \%(0 / 3)$ & $0 \%(0 / 5)$ & - & 0 & $37.5 \%(3 / 8)$ \\
\hline Total & $2 \%(2 / 99)$ & $6.4 \%(29 / 450)$ & $p=0.1728$ & 13 & $20.4 \%(112 / 549)$ \\
\hline
\end{tabular}

Table 2: Proportion of breast cancer cases with close/dirty margins by lesion distribution, disease extent, and type of surgery.

concordant with the results of some earlier publications [1] and some more recent papers indicating the suitability of breast conserving surgery in multifocal breast carcinomas [10]. Multifocality itself does not distinguish cases suitable for breast conserving surgery from those requiring mastectomy; the tissue volume that the synchronous multiple foci occupy in the breast (disease extent) is more important. Though the involved volume of the breast tissue is three-dimensional and often irregular, it is documented in two-dimensional histological slides and routinely measured in two dimensions. Although this is an obvious limitation, we previously found a 2.75 times higher relative risk of ipsilateral local recurrence in cases with a disease extent $\geq 40$ $\mathrm{mm}$ in the largest dimension than in cases with a more limited extent [12]. The results of the present study are in line with our previous findings, as differences in the frequency of dirty/close margins and mastectomy rates were much higher in extensive tumors than in nonextensive tumors.

We found significant differences in mastectomy rates between extensive and non-extensive cases among histologically unifocal tumors, in which the large extent was related to a tumor size $\geq 40 \mathrm{~mm}$. This finding was expected because mastectomy is recommended for this tumor size in regional guidelines. Differences in mastectomy rates were also found among tumors with histologically verified multiple invasive foci, but not in tumors with histologically unifocal invasive and non-unifocal in situ components. This finding may be explained by the limitation of not distinguishing lobular and ductal in situ components and in situ components by tumor grade in the present study, though the presence of lobular cancer in situ on the margin was not an indication for mastectomy according to the guidelines. Diffuse invasive breast carcinomas comprised a relatively small subgroup of tumors in the present series and were treated with mastectomy in a high proportion of cases. Generally, the mastectomy rates were similar and low in non-extensive cases (with exception of diffuse invasive tumors) and similar and high in extensive cases (with exception of unifocal invasive cancer associated with a non-unifocal invasive component) in the present series.

Both the radiological and histological tumor size reflected the expected range in a population with regular mammography screening, as approximately $70 \%$ of the tumors measured $<20 \mathrm{~mm}$. A shift towards the $10-19 \mathrm{~mm}$ category was observed in the histological measurements. Most additional invasive tumor foci in multifocal cases measured $<10$ $\mathrm{mm}$, which may explain the high proportion of radiologically occult foci in the present series. The other most frequent reason for the discrepancies in the radiological and pathological findings was that the in situ components of the tumors were calcified in only about a quarter of the low-grade and half of the high-grade cases (data not shown).

The current trend towards more and more restrictive breast cancer surgery has its background in studies demonstrating a decline in the frequency of local recurrence after breast conserving surgery [18] and a lack of benefit from margins beyond "no ink on invasive tumor or in situ cancer" $[8,19]$. Some studies have indicated that tumor characteristics, such as lobular histology, extensive intraductal component, and molecular phenotype, do not influence the local outcome [20]. In contrast, several studies have indicated a high risk of local recurrence in multifocal invasive breast carcinomas [11], extensive tumors [12], and HER2-positive cancers [21,22], which are associated with an extensive high-grade in situ component [23]. Our present study also showed a clear impact of disease extent and multifocality on margin status and mastectomy rates.

Our study has several limitations. The findings are from a regularly 
Citation: Tot T, Gere M (2016) Radiologically Unifocal Invasive Breast Carcinomas: Large-Section Histopathology Correlate and Impact on Surgical Management. J Cancer Sci Ther 8: 050-054. doi:10.4172/1948-5956.1000389

screened population in a single Swedish county. This study was also conducted at a single institution that routinely uses large-format histology slides to evaluate breast surgical specimens; therefore, the results cannot be directly compared to the vast majority of similar series based on conventional histopathology sampling. In addition, the study is purely morphological and does not analyze the impact of individual imaging methods. Furthermore, the findings cannot be related to follow-up results because this study was based on a recent series.

In conclusion, we demonstrated that $44.5 \%$ of the tumors judged as unifocal based on the pre-operative multimodal radiology workup had separate additional invasive and/or in situ foci on largeformat histopathology slides. These foci occupied an area $\geq 40 \mathrm{~mm}$ in $29.0 \%$ of cases, indicating extensive disease. Close/dirty margin(s) upon histological examination was associated with extensive disease, multifocality, and breast conservation. Thus, removing the radiologically detectable tumor focus with "no ink on the tumor" may not be sufficient in such cases.

\section{References}

1. Holland R, Veling SH, Mravunac M, Hendriks JH (1985) Histologic multifocality of Tis, T1-2 breast carcinomas. Implications for clinical trials of breastconserving surgery. Cancer 56: 979-990.

2. Tot $T$ (2012) The role of large-format histopathology in assessing subgross morphological prognostic parameters: a single institution report of 1000 consecutive breast bancer cases. Int J Breast Cancer 2012:395415

3. Mariscotti G, Houssami N, Durando M, Bergamasco L, Campanino PP, et al (2014) Accuracy of mammography, digital breast tomosynthesis, ultrasound and MR imaging in preoperative assessment of breast cancer. Anticancer Res 34: $1219-1225$

4. Sardinelli F, Giuseppetti GM, Panizza P, Bazzocchi M, Fausto A, et al. (2004) Sensitivity of MRI versus mammography for detecting foci of multifocal, multicentric breast cancer in fatty and dense breasts using whole-breast pathologic examination as a gold standard. AJR Am J Roentgenol 183: 11491157.

5. Tot T (2014) Breast cancer subgross morphological parameters and their relation to molecular phenotypes and prognosis. The Journal of Oncopathology 2: $69-76$

6. Berg WA, Gilbreath PL (2000) Multicentric and multifocal cancer: whole-breas US in preoperative evaluation. Radiology 214: 59-66.

7. Amy D, Durante E, Tot T (2015) The lobar approach to breast ultrasound imaging and surgery. J Med Ultrason 42: 331-339.

8. Coates AS, Winer EP, Goldrisch A, Gelber RD, Gnant M, et al. (2015) Tailoring therapies - improving the management of early breast cancer: St Gallen International Expert Consensus on the Primary Therapy in Early Breast cancer 2015. Ann Oncol 26:1533-1546.
9. Lynch SP, Lei X, Hsu L, Meric-Bernstam F, Buchholz TA, et al. (2013) Breast cancer multifocality and multicentricity and locoregional recurrence. Oncologist 18: $1167-1173$

10. Nijenhuis MV, Rutgers EJ (2015) Conservative surgery for multifocal/ multicentric breast cancer. Breast Suppl 2: S96-S99.

11. Chung AP, Huynh K, Kidner T, Mirzadehgan P, Sim MS, et al. (2012 Comparison of outcomes of breast conserving therapy in multifocal and unifocal invasive breast cancer. J Am Coll Surg 215: 137-146.

12. Lindquist $D$, Hellberg $D$, Tot $T$ (2011) Disease extent $\geq 4 \mathrm{~cm}$ is a prognostic marker of local recurrence in T1-2 breast cancer. Patholog Res Int 2011 860584.

13. Tot $T$ (2011) Subgross morphology, the sick lobe hypothesis, and the success of breast conservation. Int J Breast Cancer 2011: 634021.

14. http://www.cancercentrum.se/Global/RCCUppsalaOrebro/ V\%c3\%a5rdprocesser/Br\%c3\%b6stcancer/v\%c3\%a5rdprogram/ $\mathrm{Br} \% \mathrm{c3} \%$ b6stcancer\%20Regionalt_VP_2014_finalversion.pdf

15. Tot $T$ (2007) The clinical relevance of the distribution of the lesions in 500 consecutive breast cancer cases documented in large-format histological sections. Cancer 110: 2551-2560.

16. Tot T, Tabar L, Dean PB (2014) Practical Breast Pathology. (2ndedtn), Thieme, Stuttgart, New York 122-131.

17. Early Breast Cancer Trialists' Collaborative Group (EBCTCG), Darby S McGale P, Correa C, Taylor C, et al. (2011) Effect of radiotherapy after breastconserving surgery on 10-year recurrence and 15-year breast cancer death: meta-analysis of individual patient data for 10,801 women in 17 randomised trials. Lancet 378: 1707-1716.

18. Bouganim N, Tsvetkova E, Clemons M, Amir E (2013) Evolution of sites of recurrence after early breast cancer over the last 20 years: implications for patient care and future research. Breast Cancer Res Treat 139: 603-606.

19. Houssami N, Morrow M (2014) Margins in breast conservation: a clinician's perspective and what the literature tells us. J Surg Oncol 110: 2-7.

20. Moran MS, Schnitt SJ, Guliani AE, Harris JR, Khan SA, et al. (2014) Society of Surgical Oncology - American Society of Radiation Oncology consensus guidelines on margins for breast conserving surgery with whole breast irradiation in stage I and II invasive breast cancer. J Clin Oncol 32: 1507-1515.

21. Gonzalez-Angulo AM, Litton JK, Broglio KR, Meric-Bernstam F, Rakkhit R, et al. (2009) High risk of recurrence for patients with breast cancer who have human epidermal growth factor receptor 2-positive, node-negative tumors $1 \mathrm{~cm}$ or smaller. J Clin Oncol 27: 5700-5706.

22. Rouanet $P$, Roger $P$, Rousseau $E$, Thibault $S$, Romieu G, et al (2014) HER2 overexpression a major risk factor for recurrence in pT1a-bNOMO breast cancer: results from a French regional cohort. Cancer Med 3: 134-142.

23. Tot T (2015) Early ( $<10 \mathrm{~mm}$ ) HER2-positive invasive breast carcinomas are associated with extensive diffuse high-grade DCIS: Implications for preoperative mapping, extent of surgical intervention, and disease-free survival. Ann Surg Oncol 22: 2532-2539. 